Pediat. Res. 5: 274-279 (1971)

bile acids meconium newborn placenta

\title{
Primary and Secondary Bile Acids in Meconium
}

\author{
Harvey L. Sharp ${ }^{[34]}$, Janet Peller, James B. Garey, Jr., and William Krivit \\ Departments of Pediatrics and Medicine, University of Minnesota Health Sciences Center, Minneapolis, Minnesota, USA
}

\begin{abstract}
Extract
Meconium, newborn stool, and fetal bile were examined for bile acids by the techniques of gas liquid chromatography, thin layer chromatography and colorimetric spectrometry. Cholic, chenodeoxycholic, deoxycholic, and lithocholic acid were detected in meconium. Secondary bile acids were not present in significant amounts in either stool or fetal bile from newborns. During fetal development more chenodeoxycholate than cholate is formed. The presence of secondary bile acids in meconium is suggestive of maternal-to-fetal transfer via the placenta.
\end{abstract}

\section{Speculation}

The number of hydroxyl groups on the basic sterol nucleus may influence bile duct development. Excess quantities of bile acids, particularly lithocholic acid, may alter the developing fetal hepatic structure.

\section{Introduction}

Altered bile acid metabolism has been implicated in the pathogenesis of hepatic disease. Feeding lithocholate (a secondary bile acid) to animals causes hepatocellular and bile duct alterations which progress to cirrhosis [12, 18, 28]. Abnormally high lithocholate levels in serum have been found in humans with cirrhosis [6]. When these levels are lowered by cholestyramine or neomycin therapy, an improvement is noted in liver function [7]. Both primary bile acid concentrations (cholic predominating over chenodeoxycholic) are increased in the serum of infants with decreased numbers of intrahepatic bile ducts [14, 23]. Interruption of the enterohepatic bile salt circulation decreases pruritus and improves liver function.

We have studied bile acids in the human fetus and newborn to further understand normal and abnormal bile transport. These investigations have included the qualitative analysis of fetal and newborn gallbladder bile, meconium, and newborn feces by several techniques including a new solvent system to separate the taurine and glycine bile acid conjugates by thin layer chromatography. Previous investigations have not documented the presence of secondary bile acids in subjects under 1 year of age, a situation presumed to result from gut flora inadequate to alter the primary bile acids $[2,8,19]$. Thus, finding the secondary bile acids, lithocholic and deoxycholic, in sterile meconium was unexpected [22]. Their presence suggests that fetal liver can be exposed to maternal bile acids via placental transport.

\section{Materials and Methods}

Meconium, obtained from newborns in the nursery during the first $24 \mathrm{hr}$ of life, was frozen and pooled in $20 \mathrm{~g}$ batches. A total of $80 \mathrm{~g}$ were analyzed from approximately 100 newborns.

Newborn feces obtained on days 3,4 , and 5 were handled in a similar manner.

Bile from the gallbladder of 14 fetuses and 6 nonfed newborns who died in the first $24 \mathrm{hr}$ of life from 
nonhepatic causes was obtained at postmortem and diluted with 95 or $100 \%$ ethanol.

Samples of meconium and feces, thawed in $75 \mathrm{ml}$ methanol, were refluxed in $1500 \mathrm{ml} \mathrm{1:1} \mathrm{chloroform-}$ methanol for $24 \mathrm{hr}$ and then in $500 \mathrm{ml}$ acetone for 8 hr. Following evaporation under nitrogen, the sample was dissolved in $200 \mathrm{ml} 95 \%$ ethanol and refluxed in $11.2 \% \mathrm{KOH}$ solution. After evaporation of ethanol, the water phase was extracted with $200 \mathrm{ml}$ petroleum ether. Hydrolysis was accomplished with $4.5 \mathrm{~N}$ sodium hydroxide in a stainless steel bomb for $24 \mathrm{hr}$ at $130^{\circ}$. After the $\mathrm{pH}$ was adjusted to below 3.5 with concentrated $\mathrm{HCl}$, the sample was extracted three times with $125 \mathrm{ml}$ chloroform, washed with distilled water, further extracted with $125 \mathrm{ml}$ ether, and evaporated with nitrogen. The sample was mixed with a few drops 1:1 chloroform-petroleum ether in a filter aid [29] and placed on a silicic acid column [11]. Fatty acids were eluted with 1.5 liters 50:50 chloroform-ethanol. Following evaporation the bile acid fraction was placed on a column [29] and the bile acids were separated by elution with increasing proportions of benzene in petroleum ether (16). Five milliliters of benzene were added to each tube fraction; they were evaporated and the acids were titrated with $0.1 \mathrm{~N}$ sodium hydroxide with phenol red as the indicator. The acid fraction peaks were analyzed for bile acids by thin layer chromatography (TLC). Gas liquid chromatography (GLC) was performed on bile acids obtained from the TLC plates and those under the various peaks as both the methyl ester and trifluoracetyl ether derivative methyl ester. For this purpose studies were done in $1 \%$ QF on "u-shaped" columns $(1.83 \mathrm{~m} \times 0.6 \mathrm{~cm})$ using a gas chromatograph [30] with a hydrogen flame detector under the conditions of $220^{\circ}$ column temperature, $230^{\circ}$ injector influx temperature, and $240^{\circ}$ detector temperature. The sample peaks were compared with the relative retention time of the authentic bile acid derivatives. The GLC confirmation of identity was obtained by adding the same standard bile acid derivative to that of the bile acid sample.

Aerobic and anaerobic cultures were obtained on both the meconium and stool sample of the newborn.

Bile from gallbladders was studied by TLC on silica gel plates [31], by GLG, and by spectrophotometric techniques [5]. Deconjugated or free bile salts were analyzed according to the method of Eneroth [9] with the solvent system of 50:50:10 isooctane-ethyl acetateacetic acid. Conjugated bile salts were separated on TLC by solvent solution of 100:11:11, butanol-acetone-water (Fig. 1).

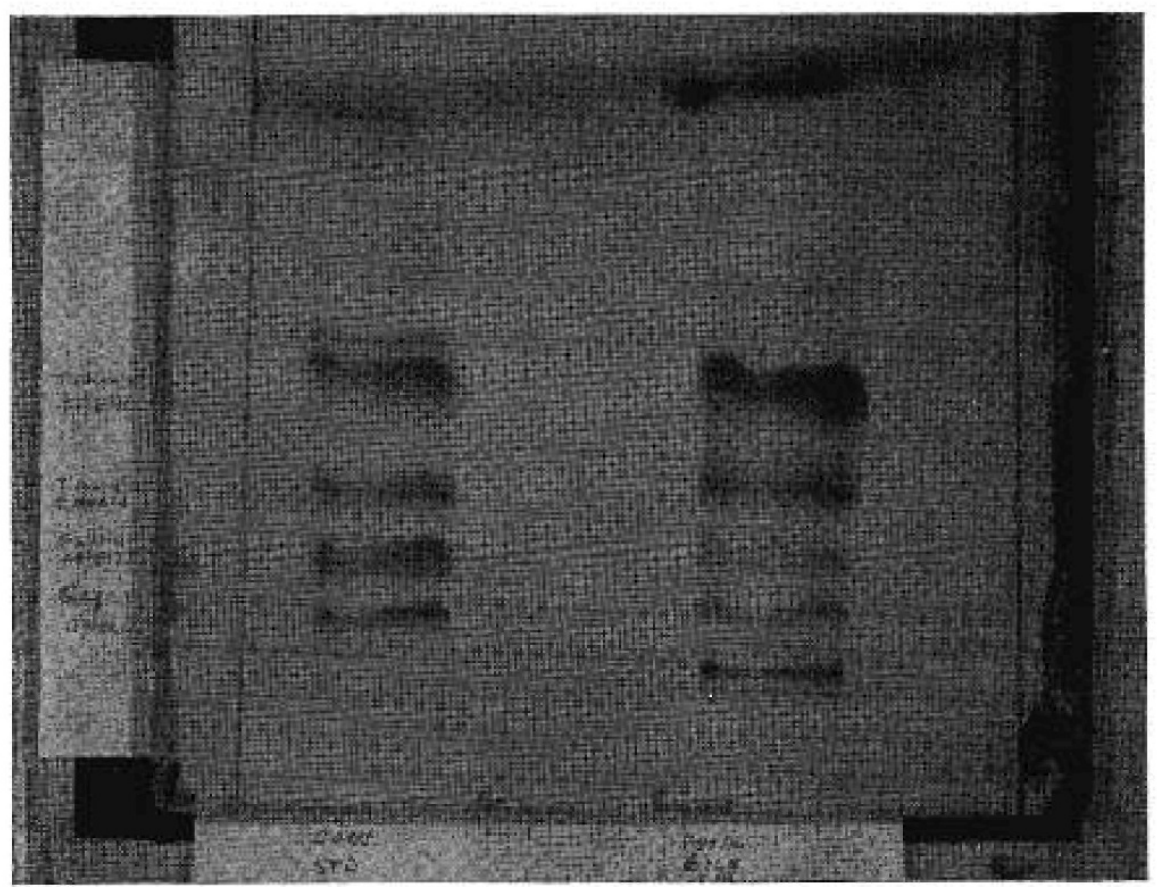

Fig. 1. Thin layer chromatography plate from a solvent system of butanol-acetone-water (100:11:11). The taurine conjugates run ahead of the glycine conjugates as shown, with the standard bile acids on the left. Bile acids from the fetal bile on the right are mainly conjugated with taurine. Note the predominance of the taurochenodeoxycholic acid. 
Results

\section{Meconium}

The meconium, cultured aerobically and anaerobically, was found to be sterile. Yet both primary and secondary bile acids could be detected by GLC and TLC. Although cholic and chenodeoxycholic acids predominated, deoxycholic acid was found in all four pooled sample batches (Fig. 2), and lithocholic acid was present in three of the four batches (Fig. 3). Verification by GLC of these bile acids was obtained by adding the appropriate standard to the sample. Only amplification of the sample peak occurred. No new peaks or basic alterations of the sample peak were

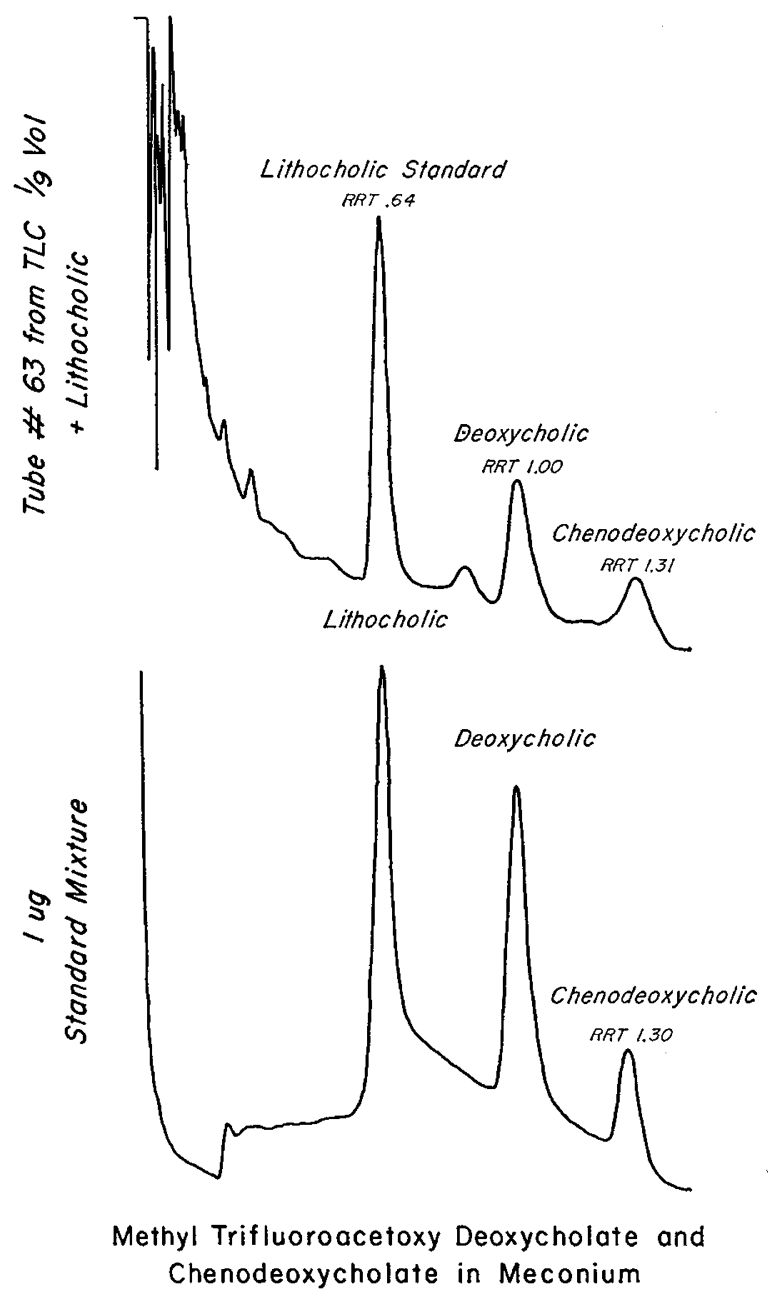

Fig. 2. Gas liquid chromatography tracings of a meconium sample (top) and a standard bile acid mixture (bottom). The relative retention time (RRT) of methyl trifluoracetoxy deoxycholate standard corresponds with the peak obtained from the meconium sample.

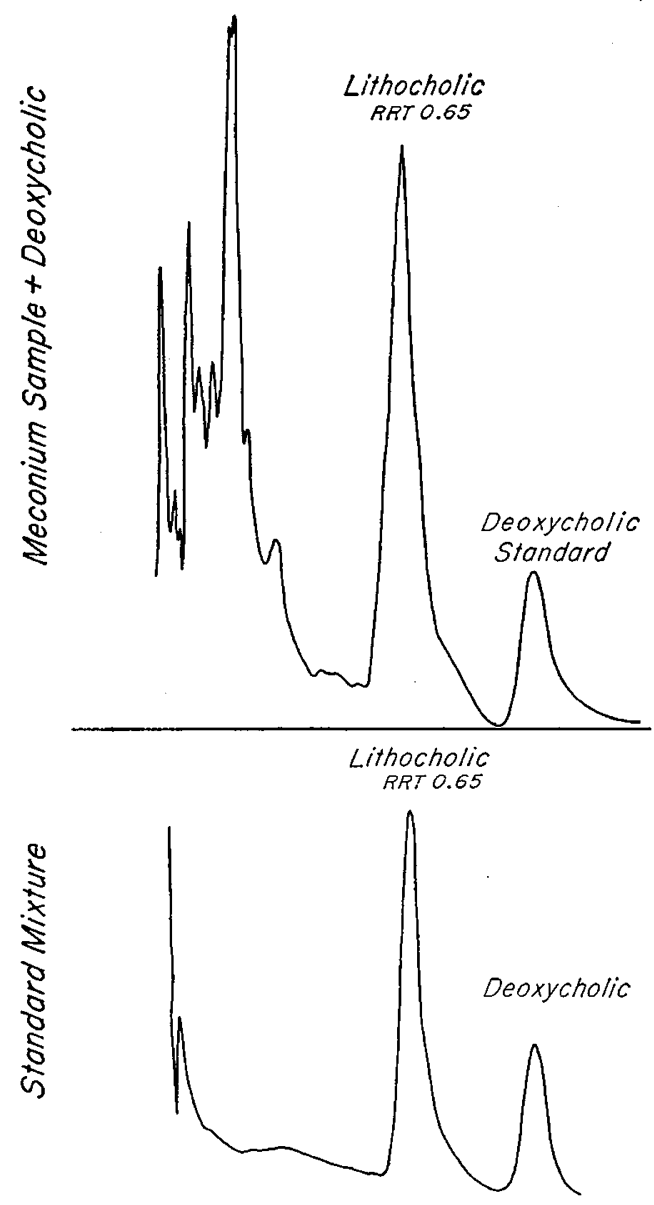

Methyl Trifluoroacetoxy Lithocholanate in Meconium

Fig. 3. Gas liquid chromatography tracings of a meconium sample (top) and a standard bile acid mixture (bottom). The relative retention time (RRT) of methyl trifluoracetoxy lithocholanate standard corresponds with the peak obtained from the meconium sample.

seen. Approximately $2.4 \mu \mathrm{g}$ lithocholic acid were present per gram of meconium when quantitation was carried out.

\section{Feces from Newborns}

No detectable secondary bile acids were found in stool samples from newborns after the first meconium passage; the first appearance occurred in analysis of feces samples from 5-day-old infants. Interestingly, the flora observed in day 5 samples included a few bacteroide species (an organism known to degrade primary bile salts into the secondary form) [l]. The amounts of both lithocholic and deoxycholic acid were no more than $0.01 \mu \mathrm{g} / \mathrm{g}$ each. 


\section{Bile}

Fetal gallbladder bile contains more chenodeoxycholic acid than cholic acid (Table I). No secondary bile acids or free bile acids were detected. Taurine conjugation predominated over glycine in all fetal samples (Fig. 1). Only in the bile of full term infants did the amount of cholic acid approximate that of chenodeoxycholic acid. At this time the quantity of bile acids was increased, but they were still mainly taurine conjugated.

\section{Discussion}

The complexity of bile acid metabolism in the fetus is diagrammatically represented in Figure 4, leaving out any consideration of fetal enterohepatic bile acid circulation for simplification purposes. Knowledge of maternal circulation is derived from previous investigations of normal adults. Studies by Carey [5] using cholorimetric techniques indicated that trihydroxy bile acids were found in greater quantity than dihydroxy bile acids. Using gas liquid chromatographic techniques, Sandberg et al. [21] found the normal serum levels of chenodeoxycholic acid higher than those of cholic acid, plus the presence of a significant quantity of deoxycholic acid. Carey [6] also found minute quantities of lithocholic acid in normal serum but did not study serum from pregnant women. The bile acids in serum, including deoxycholic acid, found during dif-
Table I. Fetal and newborn bile

\begin{tabular}{|c|c|c|}
\hline Study & $\begin{array}{c}\text { Results } \\
\text { (in order of quantity) }\end{array}$ & Methodology \\
\hline $\begin{array}{l}\text { Conjugated bile } \\
\text { acids }\end{array}$ & $\begin{array}{l}\text { 1. Taurochenodeoxy- } \\
\text { cholic } \\
\text { 2. Taurocholic } \\
\text { 3. Glycocholic } \\
\text { 4. Glycochenodeoxy- } \\
\text { cholic }\end{array}$ & $\mathrm{TLC}^{\mathbf{1}}$ \\
\hline $\begin{array}{l}\text { Bile acids after hy- } \\
\text { drolysis }\end{array}$ & $\begin{array}{l}\text { 1. Chenodeoxycholic (up } \\
\text { to } 90 \% \text { ) } \\
\text { 2. Cholic }\end{array}$ & $\begin{array}{l}\text { Spec } \\
\text { GLC } \\
\text { TLC }\end{array}$ \\
\hline Secondary bile acids & None detectable & TLG \\
\hline Free bile acids & None detectable & TLC \\
\hline
\end{tabular}

1 TLC: thin layer chromatography; Spec: colorimetric spectrometry; GLG: gas liquid chromatography.

ferent times of pregnancy did not differ from those found in the nonpregnant state [26]. Gallbladder bile from adults contains slightly more cholic than chenodeoxycholic acid with some deoxycholic but very little lithocholic acid $[4,17,25]$. These bile acids are conjugated predominantly to glycine rather than taurine, usually in a ratio of $3: 1$ [25].

We could not detect any secondary bile acids in human fetal bile (Table I), confirming the only previous study of fetal bile [19]. Other differences in the bile acid composition include the predominance of chenodeoxycholic acid over cholic acid (Table I and

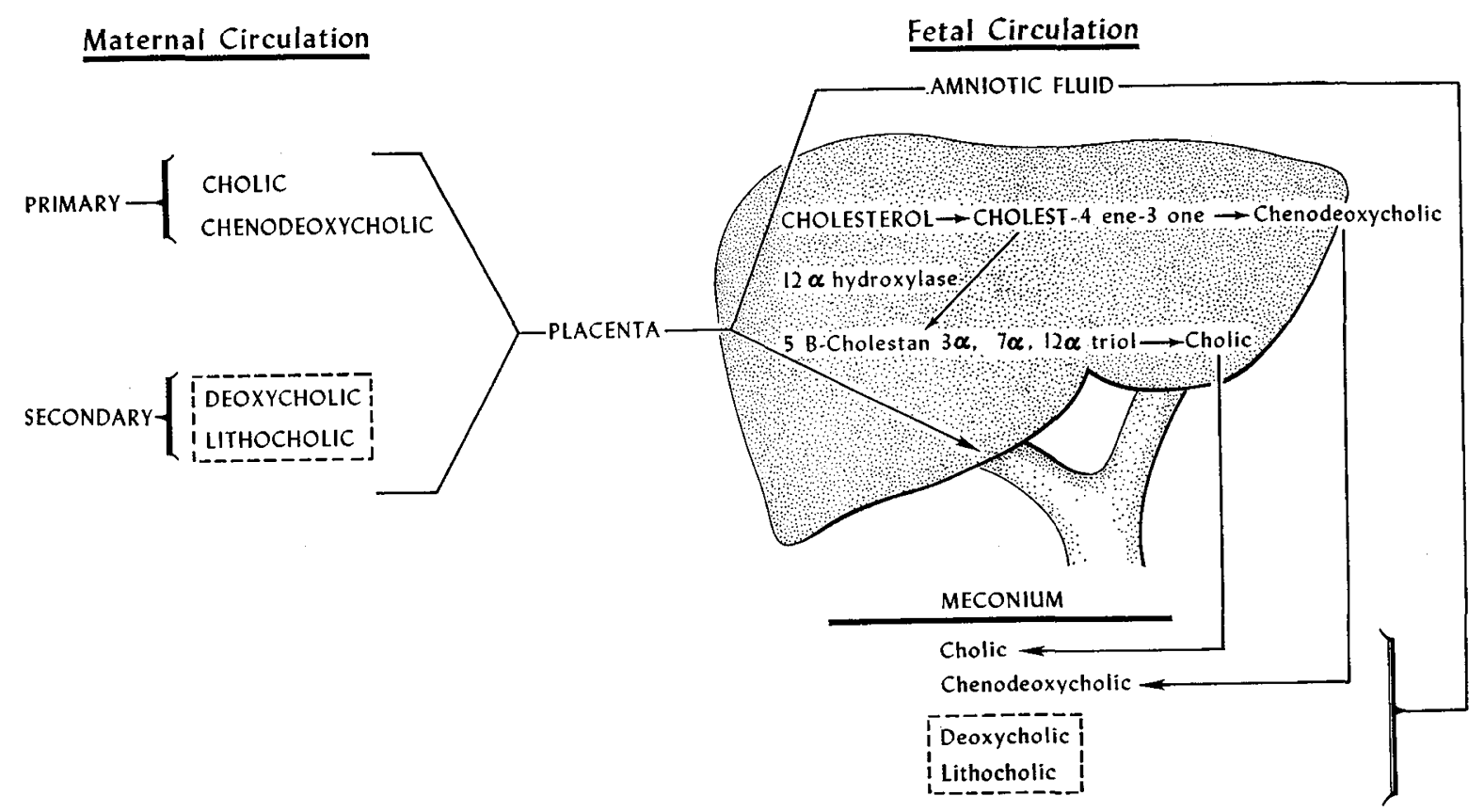

Fig. 4. Schematic proposal of maternal-fetal bile acid metabolism. 
Fig. 1). This suggests an immaturity of the 12- $\alpha$-hydroxylase enzyme system in the fetal liver, although a less likely defect in cholic acid transport is possible. Similar basic mechanisms could be proposed for the finding that taurine rather than glycine conjugation is predominant (Fig. 1). The conclusions of Poley et al. [19], based on the study of three fetal bile samples, coincide with these results. Significant changes in bile acid composition and conjugation occur during the newborn period and continue through the 1st year of life $[2,8$, $19]$.

Which bile acids are present or predominate may influence both bile duct development and infantile liver disease. The controlling factors involved in bile duct development are unknown. In animals lithocholic acid, a monohydroxy bile acid, increases the mitotic activity of the bile duct epithelium, leading to bile duct hyperplasia initially and cirrhosis eventually $[18$, 28]. Functionally, an equimolar concentration of taurocholate prevents the decreased bile flow caused by taurolithocholate administration. Pathologic elevations of predominantly cholic acid (a trihydroxy bile acid) are associated with less than the normal number of intrahepatic bile ducts in children [14, 23]. Thus it is interesting to find during bile duct development that cholic acid is not the predominant bile acid until after birth.

That significant quantities of lithocholic and deoxycholic acid (Figs. 2, 3; perforated box in Fig. 4) were found in sterile meconium was surprising. Their authenticity was documented by TLC and GLC. The same techniques used on stool samples from newborns other than meconium could not detect even minute amounts of secondary bile acids until day 5. These secondary bile acids are formed by bacterial alterations of chenodeoxycholic and cholic acid. Since meconium is sterile, we propose that these secondary bile acids are maternal in origin and pass through the placenta to the fetus.

Other supporting evidence for a maternal-to-fetal transfer of bile acids includes a preliminary report by Bongiovanni et al. [3], who confirmed by use of mass spectrometry the presence of small amounts of lithocholic acid in a 39-g sample of meconium. Also Smallwood et al. [27] recently detected secondary bile acids in the fetal bile of near-term dogs. We have made preliminary observations on human amniotic fluid and found traces of cholic, chenodeoxycholic, and deoxycholic acid but no lithocholic acid. Since the meconium studies were done from pooled samples (from about 25 newborns), it was not surprising to find varia- bility in the bile aids. Future investigation with tagged bile acids injected into maternal circulation may resolve the exact pathway of the secondary bile acids found in meconium.

Serendipitously, the substitution of acetone for acetic acid in the thin layer solvent solution more consistently separated the glycine- and taurine-conjugated primary bile acids. An unexplained finding is the unusual polar distribution of the conjugated taurine by this solvent system. Unfortunately, it does not clearly separate glycodeoxycholic from glycochenodeoxycholic acid or glycolithocholic from taurocholic acid; nevertheless, this more complete separation of primary conjugates should allow one to scrape the plates for more accurate individual quantitation.

Our serum studies of children with various forms of liver disease have failed to reveal a significant elevation of lithocholic acid. The finding of secondary bile acids in the meconium, however, does introduce the possibility of transplacental passage of these hepatotoxins during the stage of hepatic development in the fetus.

\section{Summary}

Fetal bile contains more chenodeoxycholic acid than cholic acid mainly conjugated to taurine rather than glycine. Although no secondary bile acids were found in fetal bile, meconium contained a significant quantity of the secondary bile acids, deoxycholic and lithocholic acids. Since these secondary bile acids did not persist in later newborn stools, transplacental transfer of maternal bile acids should be considered.

\section{Addendum}

The lithocholic acid peak from meconium samples identified by GLC has been further analyzed by mass spectrometry. Identical patterns were obtained, including molecular weights for standard methyl lithocholate and the sample from meconium. This confirms that the lithocholic acid peak identified by GLC in the meconium was composed of this single secondary bile acid.

\section{References and Notes}

1. Aries, V., Crowther, J. S., Drasar, B. X., and Hill, J. J.: Degradation of bile salts by human intestinal bacteria. Gut, 10 : 575 (1969).

2. Bongrovanni, A. M.: Bile acid content of gallbladder in children and adults. J. Clin. Endocrinol. Metab., 25: 678 (1965).

3. Bonglovanni, A. M., Eberlein, W. R., and Marino, J.: 
Steroids in meconium. Read by title at 79 th Annual Meeting of the American Pediatric Society, p. 64, 1969.

4. BuRnert, W.: The pathogenesis of gallstones. In: W. Taylor: The Biliary System, p. 601 (F. H. Davis Company, City, 1965).

5. CAREY, J. B., JR.: Serum trihydroxy-dihydroxy bile acid ratio in liver and biliary diseases. J. Clin. Invest., 37: 1494 (1958).

6. Carex, J. B., JR., AND Wlliams, G.: Lithocholic acid in human serum. Science, 150: 620 (1965).

7. Carey, J. B., JR., Wilson, F. D., Onstad, G., and Zaki, F. C.: Role of 12 alpha hydroxylose deficiency in continuing liver injury. J. Clin. Invest., 46: 1042 (1967).

8. EnCRantz, J. C., and SJovall, J.: On the bile acids in duodenal contents in infants and children. Clin. Chim. Acta, 4: 793 (1959).

9. ENERoTh, P.: Thin layer chromatography of bile acids. J. Lipid Res., 4: 11 (1963).

10. From, S., AND WAGNER, H.: Quantitative determination of conjugated bile acids in serum in acute hepatitis. Nature, 213: 404 (1967).

11. Hirsch, J., AND Ahrens, E. H., JR.: The separation of complex lipid mixtures by the use of silicic acid chromatography. J. Biochem., 233: 311 (1965).

12. Holsti, P.: Cirrhosis of the liver induced in rabbits by gastric instillation of 3-monohydroxycholic acid. Nature, 186: 250 (1960).

13. JavitT, N. B.: Cholestasis in rats induced by taurolithocholate. Nature, 210: 1262 (1966).

14. Lottsfeldt, F. I., Krivit, W., Aust, J. B., And Carey, J. B., JR.: Cholestyramine therapy in intrahepatic biliary atresia. New Engl. J. Med., 269: 186 (1963).

15. McLeod, G. M., AND Wiggins, H. S.: Bile salts in small intestinal contents after ileal resection and other malabsorption syndromes. Lancet, $i: 873$ (1968).

16. Mosbach, E. H., Zomzley, C., And Kendall, F. E.: Separation of bile acids by column-partition chromatography. Arch. Biochim. Biophys., 48: 95 (1954).

17. Nakayami, F.: Quantitative microanalysis of bile. J. Lab. Clin. Med., 69: 594 (1967).

18. Palmer, R. H., and Hruban, Z.: Production of bile duct hyperplasia and gallstones by lithocholic acid. J. Clin. Invest., 45: 1255 (1966).
19. Poley, J. R., Power, J. G., Owen, C. H., JR., and Stickler, G. B.: Bile acids in infants and children. J. Lab. Clin. Med., 63: 838 (1964).

20. Ryhage, R., and Stenhagen, E.: Mass spectrometry in lipid research. J. Lipid Res., 1: 361 (1959).

21. Sandberg, D. H., Sjovall, K., And Turner, D. A.: Measurement of human serum bile acids by gas-liquid chromatography. J. Lipid Res., 6: 182 (1965).

22. Sharp, H. L., Carey, J. B., Jr., Peller, J., and Krivit, W.: Lithocholic acid in meconium. Pediat. Res., 2: 293 (1968).

23. Sharp, H. L., Carey, J. B., Jr., White, J. G., and Krivit, W.: Cholestyramine therapy in patients with a paucity of intrahepatic bile ducts. J. Pediat., 71: 723 (1967).

24. Shiodu, R., Wood, P. D. S., ANd Kinsell, L. W.: Determination of individual conjugated bile acids in human bile. $\mathrm{J}$. Lipid Res., 10: 546 (1969).

25. Sjovall, J.: Bile acids in man under normal and pathological conditions. Clin. Chim. Acta, 5: 33 (1960).

26. Sjovall, K., AND Sjovall, J:: Serum bile acid levels in pregnancy with pruritis. Clin. Chim. Acta, 13: 207 (1966).

27. Smallwood, R. A., Lester, R., Piasichi, G. J., Helmut, F. J., AND JACKSON, B. T.: Metabolism of bile salt in the fetal dog. Presented at the 16th Annual Meeting of the American Society for Clinical Investigation, p. 78, 1969.

28. Zaki, F. G., Carey, J. B., JR., Hoffbauer, F. W., and NwokoLo, C.: Biliary reaction and choledocholithiasis induced in the rat by lithocholic acid. J. Lab. Clin. Med., 65: 737 (1967).

29. Celite, Johns-Manville Products Corporation, New York, N. Y.

30. Model 5000, Barber-Colman Company, Rockford, Ill.

31. Silica gel G, E. Merkag, Brinkmann Instruments, Inc., Great Neck, N.Y.

32. Presented in part at the Meeting of the Society for Pediatric Research, May, 1968, Atlantic City, N. J.

33. Supported by St. Mary's Marion Research Foundation; by Public Health Service Research Grants nos. CA 08832, CA 08181, and CA 07306; and by the Graduate School of the University of Minnesota.

34. Requests for reprints should be addressed to: Harvey $\mathbf{L}$. Sharp, M.D., Department of Pediatrics, University of Minnesota Health Sciences Center, Minneapolis, Minn. 55455 (USA).

35. Accepted for publication August 28, 1970. 